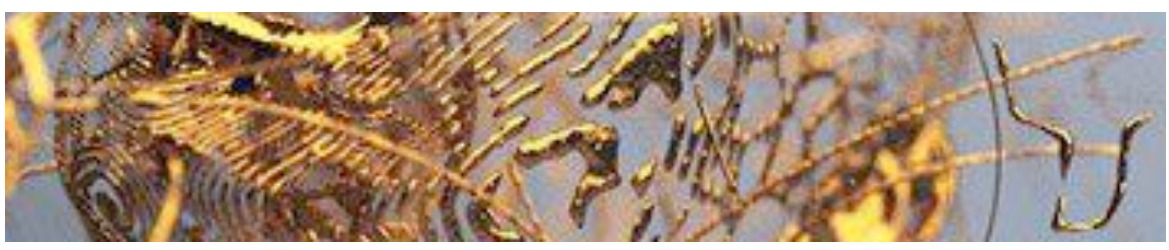

\title{
Derechos fundamentales y mediación en la Unión Europea: hacia un enfoque de derechos
}

\section{Fundamental rights and mediation in the European Union: a new approach of rights}

Malagón Terrón, Francisco Javier ${ }^{(*)}$

Universidad Complutense de Madrid - Madrid

fjmalagon@ccinf.ucm.com

\section{Resumen}

El autor propone un "enfoque de derechos" para reorientar las prácticas de Mediación en Conflictos de modo que, mediante modelos de actuación preventivos y de respuesta temprana, evite el agravamiento de los conflictos, contribuyendo con ello a la preservación de los Derechos Fundamentales de los ciudadanos.

Palabras clave: Mediación, conflictos, derechos fundamentales, prevención, respuesta temprana, equidad.

\section{Abstract}

The author proposes a "human rights approach" to redirect Conflict Mediation practices so that, through models of preventive action and early response, avoid the escalation of conflicts, thus contributing to the preservation of Fundamental Rights citizens.

Keywords: Mediation, conflict, fundamental rights, prevention, early response, equity. 


\section{CONFLiCTO Y DERECHOS FUNDAMENTALES}

Los conflictos de las sociedades europeas, consecuencia de la crisis del Antiguo Régimen y del desarrollo de la industrialización, encontraron en el siglo XX un elemento pacificador y de regulación en el Estado social y democrático de Derecho.

En esta forma avanzada de Estado los derechos de los ciudadanos fueron objetivados como derechos fundamentales, expresión que se refiere a los presupuestos del consenso sobre el que debe edificarse cualquier sociedad democrática; esto es: al sistema de valores y principios de carácter universal que informan el ordenamiento jurídico de nuestras sociedades ${ }^{1}$.

Existe, por tanto, una relación consustancial entre el Estado de Derecho y los derechos fundamentales los cuales reconocen y garantizan tanto las libertades individuales como los derechos de carácter económico, social y cultural. Estos derechos han sido proclamados por el Parlamento Europeo, el Consejo Europeo y la Comisión Europea en el año 2000 en declaración solemne mediante la Carta de los Derechos Fundamentales de la Unión Europea. ${ }^{2}$

La protección de estos derechos ha encontrado en los sistemas de Justicia un instrumento fundamental, junto con los otros poderes del Estado y la acción autónoma de los ciudadanos en el ejercicio de sus libertades civiles; sin embargo, actualmente los sistemas de Justicia parecen adolecer de importantes limitaciones para llevar a cabo su función garantista de manera eficaz y, sobre todo, de modo satisfactorio para los ciudadanos.

\section{LA MEDIACIÓN COMO OPORTUNIDAD}

La Mediación, entendida como práctica específica de gestión negociada de conflictos, se ha ido implantando en los países de la Unión Europea a lo largo de los años 90 y 2000, si bien de forma muy desigual según los

\footnotetext{
${ }^{1}$ Cf. Pérez Luño, Antonio E. (1984): Los derechos fundamentales. Madrid: Ed. Técnos.

2 Carta de los Derechos Fundamentales de la Unión Europea (2000/C 364/01), publicada en el Diario Oficial de las Comunidades Europeas el 18.12.2000.
} 
países, con el propósito declarado de complementar a los sistemas de Justicia tradicionales.

La Mediación es un proceso en el que un experto imparcial (es decir, que no se posiciona en contra de ninguna de las partes) y neutral (que respeta la autonomía de los mediados para producir sus propios acuerdos) facilita la comunicación entre dos o más personas con el fin de ayudarles a componer sus propios acuerdos, dentro de los derechos y límites que en cada momento les reconocen las leyes.

Si bien a esta forma de mediación ${ }^{3}$ cabe encontrarle antecedentes muy antiguos, es desde los años 70 del pasado siglo que se viene configurando como un campo profesional específico, incluido dentro de un campo más amplio de procedimientos extrajudiciales de solución de disputas conocido como Resolución Alternativa de Conflictos (Alternative Dispute Resolution, $A D R)$, el cual incluye, además, otras técnicas como la Negociación, el Arbitraje y la Conciliación.

Básicamente, son tres los argumentos que suelen aducirse para justificar el interés social por la Mediación: a) su rapidez y bajo coste, en comparación con los procedimientos litigiosos; b) la satisfacción que reporta a los ciudadanos, en tanto que les permite auto-componer de forma negociada soluciones a sus conflictos; y c) el desahogo que reporta a los sistemas de Justicia al reducir el número y la duración de los procesos judiciales.

La Unión Europea viene impulsando a lo largo del nuevo siglo la regulación e implantación de esta forma de Mediación en los Estados miembros. A tal efecto, la Comisión elaboró en 2002 un Libro Verde sobre las modalidades alternativas de solución de conflictos en el ámbito del derecho civil y mercantil, en el que hacía balance de la situación imperante en lo que respecta a métodos de solución de conflictos en la Unión Europea y con el que inició una amplia consulta con los Estados miembros y las partes interesadas sobre posibles medidas para promover la Mediación.

\footnotetext{
${ }^{3}$ Conviene recordar que, de facto, la palabra mediación es una categoría general de uso extendido en muchos ámbitos de la acción y del conocimiento humano (Filosofía, Religión, Sociología, Economía, Comunicación, etc.). Aquí hacemos referencia a una forma específica de mediación que, en las últimas décadas, a adoptado, además, una forma profesional.
} 
Como consecuencia de ese proceso de reflexión, en 2008 fue aprobada la Directiva 2008/52/CE del Parlamento Europeo y del Consejo de 21 de mayo de 2008 sobre ciertos aspectos de la mediación en asuntos civiles y mercantiles ${ }^{4}$, norma que informa y delimita la legislación sobre Mediación de los Estados miembros.

Sin embargo, es un hecho ampliamente reconocido que los ciudadanos europeos tienen aún un escaso conocimiento de la Mediación, que la implantación de servicios y dispositivos de Mediación es aún incipiente en la mayor parte de los países y que las organizaciones relacionadas específicamente con la Mediación (asociaciones profesionales, empresas...) adolecen de debilidades estructurales notorias y de un mercado profesional reducido y precario.

En este sentido se ha pronunciado en febrero de 2014 el Comité de Asuntos Legales del Parlamento Europeo, el cual ha analizado las causas del escaso éxito de la Mediación en Europa en el estudio que lleva por título "Reiniciar la directiva de mediación: evaluando el limitado impacto de su Implementación y proponiendo medidas para incrementar el número de mediaciones en la Unión Europea”.

En el marco de este estudio un grupo de reconocidos expertos internacionales en mediación y arbitraje han desarrollado la idea de la "Paradoja de la Mediación en la Unión Europea" para señalar que a pesar del impulso que esta práctica profesional ha recibido por parte del Consejo de Europa, la Comisión Europea y numerosos gobiernos, únicamente ha sido utilizada el año pasado en un $1 \%$ de los litigios civiles y mercantiles surgidos en Europa.

En sus conclusiones, el grupo de expertos propone "reiniciar" (haciendo un símil con el lenguaje informático) la Directiva 2008/52/CE mediante una modificación a fondo de la norma o mediante un compromiso

\footnotetext{
${ }^{4}$ Cf. Directiva 2008/52/CE del Parlamento Europeo y del Consejo de 21 de mayo de 2008 sobre ciertos aspectos de la mediación en asuntos civiles y mercantiles, publicada en el Boletín Oficial de las Comunidades Europeas el 24.5.2008.

${ }^{5}$ Estudy "Rebooting the mediation directive: assessing the limite impact of its implementation and proposing measures to increase the number of mediations on UE" (Directorate General for Internal Policies. Policy Department C: Citizen's Rights and Constitutional Affairs, 2014). Disponible en: http://www.europarl.europa.eu/RegData/etudes/etudes/join/2014/493042/IPOLJURI_ET(2014)493042_EN.pdf
} 
de cada Estado que le obligue a conseguir una relación equilibrada entre el proceso judicial y la mediación. Entre otras medidas, sugieren también acciones de sensibilización, el desarrollo de proyectos piloto e, incluso, la introducción de la obligatoriedad de la Mediación por categorías de asuntos o áreas de conflictividad.

\section{HACIA UN ENFOQUE DE DERECHOS}

Proponer un "enfoque de derechos" para la Mediación es una forma de contribuir a la superación de algunas de las limitaciones que actualmente adolece el sector, mediante la articulación de dos perspectivas jurídicas:

a) el derecho de los ciudadanos a una red capilar de servicios de Mediación, accesibles, suficientes y de calidad, para prevenir y gestionar con autonomía y de forma temprana los conflictos que les afec$\tan ; \mathrm{y}$

b) la Mediación como uno de los recursos estratégicos que el Estado democrático Social y de Derecho debe desplegar para la protección de los derechos fundamentales de sus ciudadanos, tanto en el plano de sus libertades civiles como de sus derechos sociales, económicos y culturales.

Los derechos de los ciudadanos se ven más amenazados cuanto más graves son los conflictos que les afectan; y los diversos conflictos interpersonales y grupales tienden a agravarse cuanto mayores son las desigualdades sociales.

Por este motivo la Mediación, entendida desde un enfoque de derechos, es un conjunto de modelos y prácticas que pueden contribuir a reducir las brechas entre los ciudadanos, que son, en buena medida, consecuencia de las desigualdades crecientes que experimentan las sociedades europeas.

No se trata sólo de actuar sobre el sistema Judicial, para mejorarlo o descargarlo de trabajo gracias a la Mediación, sino de intervenir en la misma dinámica social de la que emergen los conflictos, favoreciendo que las prácticas mediadoras contribuyan al ajuste negociado y equitativo de los intereses de los ciudadanos. 
Pero no se trata sólo de ofrecer servicios de Mediación cuando los conflictos se han agravado y están a punto de judicializarse, sino en el origen de las relaciones y de forma preventiva, contribuyendo a fundar vínculos de convivencia y/o colaboración sostenibles; así como mediante prácticas que aportan respuestas mediadoras tempranas cuando los conflictos aparecen.

Desde este enfoque de derechos, la Mediación no es una mera opción para el Estado, sino una obligación que debería garantizar, como lo hace con el acceso a la educación o a la sanidad públicas, como recursos imprescindibles para que la ciudadanía pueda experimentar el bienestar de pertenecer a una sociedad más equitativa, democrática e integradora.

\section{Capilaridad y diversidad de MOdelos Y DE ACTORES}

Un enfoque de derechos como el que se propone requiere que la Mediación se promueva capilarmente; esto es, de manera que sea un recurso conocido, cercano y accesible para todos los ciudadanos. Sin duda Internet y las modernas tecnologías de la comunicación pueden contribuir a ello, siempre que demuestren ser medios complementarios eficaces y no meros sustitutivos baratos de otras formas de acceso a las prácticas mediadoras.

Ahora bien, no es suficiente con que la Mediación sea un servicio aparentemente cercano, gratuito o de bajo coste si no se tiene en cuenta, además, que la manera de mediar tiene que estar adaptada a las características de los distintos conflictos, pero también de los distintos grupos de población y de sus contextos de vida.

Finalmente, este enfoque de derechos requiere de un nuevo posicionamiento del profesional de la Mediación, de modo que su relevancia y su eficacia resida, por supuesto, en su intervención directa, pero también en su capacidad para aportar criterios y competencias mediadoras al resto de la población. El derecho de los ciudadanos a la Mediación no se limitaría, por tanto, a ser sus sujetos mediados, sino también a ser mediadores competentes en su contexto. 


\section{Bibliografía}

Carta de los Derechos Fundamentales de la Unión Europea (2000/C 364/01). Publicada en el Diario Oficial de las Comunidades Europeas el 18.12.2000.

Directiva 2008/52/CE del Parlamento Europeo y del Consejo de 21 de mayo de 2008 sobre ciertos aspectos de la mediación en asuntos civiles y mercantiles. Publicada en el Boletín Oficial de las Comunidades Europeas el 24.5.2008.

Directorate General for Internal Policies. Policy Department C: Citizen's Rights and Constitutional Affairs (2014): "Rebooting the mediation directive: assessing the limite impact of its implementation and proposing measures to increase the number of mediations on UE”. Disponible en:

http://www.europarl.europa.eu/RegData/etudes/etudes/join/2014/493042/IP OL-JURI_ET(2014)493042_EN.pdf

PÉREZ LuÑo, A. E. (1984): Los derechos fundamentales. Madrid: Ed. Técnos. 


\section{PARA CITAR ESTE TRABAJO EN BIBLIOGRAFÍAS:}

MALAGÓN TERRÓN, Francisco Javier (2013): "Derechos fundamentales y mediación en la Unión Europea: hacia un enfoque de derechos", Mediaciones Sociales. Revista de Ciencias Sociales y de la Comunicación, $\mathrm{n}^{\mathrm{o}}$ 12, pp. 218-226. DOI: http://dx.doi.org/10.5209/rev_MESO.2013.n12.45269

\section{${ }^{(*)} \mathbf{E}$ autor}

Francisco Javier Malagón Terrón es investigador y profesor asociado de la Universidad Complutense de Madrid (Facultad de Ciencias de la Información, Departamento de Sociología IV). Es D.E.A. en Filosofía, Licenciado en Ciencias de la Información por la UCM, Máster en Mediación por la Universidad de Alcalá de Henares, Diplomado en Mediación y Resolución de Conflictos por la Universitat Oberta de Catalunya y Experto en Intervenciones Sistémicas con Familias y Organizaciones por la Escuela Vasco Navarra de Terapia Familiar. Cuenta además con una amplia formación en el ámbito de los estudios sobre las religiones, con diversos diplomas de la Universidad Complutense de Madrid, la Universidad de Alcalá y la Fundación Zubiri. Cuenta también con otros títulos relacionados con la Comunicación, la Educación, el Desarrollo, la Gestión de la Calidad y la Innovación. Su trayectoria profesional a lo largo de veinticinco años le ha permitido conocer de cerca el mundo asociativo y de las ONGs, de los ayuntamientos, la universidad, los sindicatos, los partidos políticos y las empresas. Sus aportaciones al ámbito de la Mediación en Conflictos tienen como marco de referencia una variada formación humanista e interdisciplinar, así como también un rico conjunto de prácticas profesionales susceptibles de ser analizadas y comprendidas desde el paradigma de la Mediación, siendo una de las más importantes la coordinación de redes sociales de colaboración. Actualmente, sigue compaginando su actividad académica con la actividad profesional en calidad de socio fundador y director de Entrenadores de Pensamiento, empresa de consultoría para el desarrollo de la inteligencia social y la innovación en las organizaciones.

RECIBIDO: 08 de julio de 2013.

ACEPTADO: 13 de octubre de 2013.

Mediaciones Sociales, № 12, 2013, pp. 218-226. ISSN electrónico: 1989-0494. DOI: http://dx.doi.org/10.5209/rev_MESO.2013.n12.45269 
